\title{
The Effects of Confinement on Neuropsychiatric Symptoms in Alzheimer's Disease During the COVID-19 Crisis
}

Claire Boutoleau-Bretonnière ${ }^{\mathrm{a}, \mathrm{b}}$, Hélene Pouclet-Courtemanche ${ }^{\mathrm{a}, \mathrm{b}}$, Aurelie Gillet ${ }^{\mathrm{a}}$, Amelie Bernard $^{\mathrm{a}, \mathrm{b}}$, Anne Laure Deruet ${ }^{\mathrm{a}, \mathrm{b}}$, Ines Gouraud ${ }^{\mathrm{a}, \mathrm{b}}$, Aurelien Mazoue ${ }^{\mathrm{b}}$, Estelle Lamy ${ }^{\mathrm{a}, \mathrm{b}}$, Laetitia Rocher, ${ }^{\mathrm{a}, \mathrm{b}}$, Dimitrios Kapogiannis ${ }^{\mathrm{c}}$ and Mohamad El Haj ${ }^{\mathrm{d}, \mathrm{e}, \mathrm{f}, *}$

${ }^{a}$ CHU Nantes, Inserm CICO4, Nantes, France

${ }^{\mathrm{b}}$ CHU Nantes, Départementde Neurologie, Centre Mémoire de Ressources et Recherche, Nantes, France

${ }^{\mathrm{c}}$ Laboratory of Clinical Investigation, National Institute on Aging, Baltimore, MD, USA

${ }^{\mathrm{d}}$ Nantes Université, Univ Angers, Laboratoire de Psychologie des Pays de la Loire (LPPL - EA 4638), Nantes, France

${ }^{\mathrm{e}}$ Unité de Gériatrie, Centre Hospitalier de Tourcoing, Tourcoing, France

${ }^{\mathrm{f}}$ Institut Universitaire de France, Paris, France

\begin{abstract}
.
Background: Neuropsychiatric symptoms, such as depression, anxiety, apathy, agitation, and hallucinations, are frequent in Alzheimer's disease (AD) and their prevalence tends to increase with external stressors.

Objective: We offer the first investigation of the effects of confinement during the COVID-19 crisis on neuropsychiatric symptoms in patients with AD.

Methods: We contacted caregivers of 38 patients with AD who were confined to their homes for nearly two months and asked them to report whether patients experienced any change in neuropsychiatric symptoms during, compared to before, the confinement and rate its severity and impact on themselves using the Neuropsychiatric Inventory-Questionnaire.

Results: Among the 38 patients, only 10 demonstrated neuropsychiatric changes during the confinement. Cognitive function of these 10 patients, assessed with the Mini-Mental State Examination, was worse than that of patients who did not demonstrate neuropsychiatric changes. Interestingly, among the 10 patients with neuropsychiatric changes, the duration of confinement significantly correlated with the severity of symptoms as well as with their caregivers' distress.

Discussion: The confinement seems to impact neuropsychiatric symptomatology in AD patients with low baseline cognitive function.
\end{abstract}

Keywords: Alzheimer's disease, confinement, COVID-19, neuropsychiatric symptoms

Because of their cognitive and functional impairments, patients with Alzheimer's disease (AD) are vulnerable during crises, and this has been especially

\footnotetext{
*Correspondence to: Mohamad El Haj, Faculté de Psychologie, LPPL - Laboratoire de Psychologie des Pays de la Loire, Université de Nantes, Chemin de la Censive du Tertre, BP 81227, 44312 Nantes Cedex 3, France. E-mail: mohamad.elhaj@univnantes.fr.
}

true during the corona virus (COVID-19) pandemic. To cope with the pandemic and to limit the spread of infections, governments in North America and Europe (including France) have been obliged to impose a variety of "lockdown" measures. In France, beginning on March 17, 2020, people were confined to their homes and were only permitted to leave to buy essential goods, briefly exercise, or seek medical 
help. The lockdown was partially lifted on May 11, 2020. While this lockdown has dramatically changed most people's daily lives, its impact may have been particularly pronounced in patients with AD. The social distancing measures and diminished physical contact with family and the outside world might have increased loneliness and impacted mental health among patients with $\mathrm{AD}$ who remained confined to their homes for nearly two months. Moreover, even low-risk outdoors activities, such as enjoying a walk with their spouses, were limited. Social activities (e.g., attending neighborhood meetings), religious activities (e.g., participating in mass), or even wellbeing and grooming activities (e.g., having a haircut) were canceled. To investigate whether these social and physical restrictions impacted the mental health of patients with $\mathrm{AD}$, we evaluated neuropsychiatric symptomatology of patients with AD who were confined to their homes during the COVID-19 crisis in France.

To better understand the effects of the COVID19-related confinement on neuropsychiatric symptomatology of $\mathrm{AD}$, we should be mindful of the rich neuropsychiatric symptomatology of AD. While $\mathrm{AD}$ is mainly associated with memory decline [1, 2], the disease is also associated with a wide range of neuropsychiatric symptoms [3-5] and more than $80 \%$ of patients with $\mathrm{AD}$ exhibit at least one neuropsychiatric symptom over the course of the disease [6]. Neuropsychiatric symptoms typically fluctuate in intensity over the course of $\mathrm{AD}$ [7]. While neuropsychiatric symptoms are thought to emerge in patients with more advanced $\mathrm{AD}$, these symptoms can also manifest in early $\mathrm{AD}$ or even during its prodromal phase [3, 8-11]. Research has demonstrated that early neuropsychiatric symptoms predict a faster progression from mild cognitive impairment to AD [12-14]. Even among cognitively normal older adults, the emergence of neuropsychiatric symptoms predict $\mathrm{AD}$ [15-18] and may bea better predictor of cognitive impairment than even hippocampal atrophy Geda et al. [19].

Neuropsychiatric symptoms in AD include depression, anxiety, apathy, verbal and physical agitation, and hallucinations [3, 10]. The categorization of neuropsychiatric symptoms is, however, complicated [20], probably because their nosology does not always reflect pathophysiology [11]. Although the classification system of the Diagnostic and Statistical Manual remains a point of reference, the neuropsychiatric symptoms in AD overlap substantially among differently classified disease entities.
For instance, agitation in AD may accompany anxiety, depression, or hallucinations. A different way of classifying neuropsychiatric symptoms can be based on their temporal order of occurrence. According to Masters et al. [21], neuropsychiatric symptoms in $\mathrm{AD}$ typically occur in three phases. The first phase includes depression, irritability, and nighttime behavior changes. The second phase includes anxiety, agitation, apathy, and appetite changes. The third phase includes delusions, hallucinations, disinhibition, and motor disturbances. Another classification for neuropsychiatric symptoms in $\mathrm{AD}$ was proposed by Ismail et al. [22] who classified these symptoms into five categories: drive and motivation, emotional dysregulation, impulsivity, social inappropriateness, and abnormal perceptions or thoughts. To overcome the classification limitation of neuropsychiatric symptoms in AD, Fischer et al. [23] suggested that any classification system should address their overlap with related conditions (especially, regarding agitation), recognize that symptoms may emerge in the preclinical and prodromal phases, and add a more specific differentiation between delusions and hallucinations. Regardless of their classification, neuropsychiatric symptoms in AD have been captured by the Neuropsychiatric Inventory-Questionnaire (NPIQ) [24] for decades. This inventory is widely used to assess frequency and severity of the main neuropsychiatric symptoms in $\mathrm{AD}$, namely, delusions, hallucinations, agitation, depression, anxiety, euphoria, apathy, disinhibition, irritability, aberrant motor activity, night-time behavior disturbance, and appetite disorders.

The occurrence of neuropsychiatric symptoms in $\mathrm{AD}$ accelerate the rate of disease progression and institutionalization and may affect treatment responses and prognosis [6]. Neuropsychiatric symptoms in $\mathrm{AD}$ also decrease the patients' quality of life and increase their distress [11]. Critically, neuropsychiatric symptoms increase distress in caregiver who typically struggle to manage the behavioral consequences of these symptoms [25, 26]. Considering the consequences of neuropsychiatric symptoms of $\mathrm{AD}$ patients on their caregivers, we sought to assess, not only changes in neuropsychiatric symptoms of $\mathrm{AD}$ patients during the COVID-19 crisis, but also their consequences on caregivers.

In the present study, we investigated whether the occurrence and severity of neuropsychiatric symptoms increased during the confinement. We conducted telephone interviews with caregivers (e.g., spouses, children) of patients with $\mathrm{AD}$ who were 
followed by the Memory Center of the Hospital of Nantes, France, and asked them to report on the patients' neuropsychiatric symptoms during the confinement. More specifically, we invited caregivers to indicate whether patients had manifested any changes in the occurrence and/or severity of neuropsychiatric symptoms during the confinement. When caregivers indicated that changes had occurred, we invited them to answer the questions of the NPIQ, which assess specific neuropsychiatric symptoms and the care givers' distress produced by these symptoms. We hypothesized that a positive correlation between the duration of confinement and scores on NPIQ items may be observed (i.e., the longer the confinement, the worse the symptoms), as well as a positive correlation with care givers' distress (i.e., the longer the confinement, the higher the level of distress).

\section{METHODS}

\section{Participants}

The study included 38 participants (23 women; $M$ age $=71.89$ years, $S D=8.24$ ) with a clinical diagnosis of probable $\mathrm{AD}$ and had a recent visit (two to four months prior to the study) to the Memory Center of the Hospital of Nantes-France. A diagnosis of probable $\mathrm{AD}$ had been made prior to the study by neurologists at the Memory Center according to the clinical criteria of the National Institute on Aging and the Alzheimer's Association criteria for probable AD [1]. During their last visit, the patients had been assessed with the Mini-Mental State Examination [27] $(M=18.84 / 30$ points, $S D=6.89)$. The study also included the patients' caregivers $(M$ age $=68.43$ years, $S D=12.16$ ). While most of the caregivers were the patients' spouses $(n=32)$, five were their daughters and one was a sister of a patient. The patients were living with their caregivers, in other words, none was living alone. This study was designed and conducted in accordance with the Declaration of Helsinki.

\section{Procedures}

During the confinement, more precisely between March 26, 2020, and May9, 2020, neurologists from the Memory Center of the Hospital of Nantes contacted the caregivers by phone to assess the neuropsychiatric function of the patients. Caregivers were asked to provide the exact date at which they were confined to their homes, since while the offi- cial lockdown in France started on March 17, 2020, some people followed confinement guidelines earlier or even later than this date. Caregivers were asked if they had noticed any behavioral or emotional changes in the patients during, compared with before, the confinement compared. If the answer was "yes", caregivers were invited to answer the NPIQ questions.

We implemented the NPIQ, as this scale is widely used for the assessment of neuropsychiatric symptoms in $\mathrm{AD}$. We also used the scale because it is based on interviewing care givers and it was possible to administer it over the phone during the lockdown. During the course of administering the NPIQ, we asked caregivers to rate the severity of twelve symptoms: delusions, hallucinations, agitation, depression, anxiety, euphoria, apathy, disinhibition, irritability, aberrant motor activity, night-time behavior disturbance, and appetite disorders. Severity was rated on a three-point scale (one point $=$ mild, i.e., noticeable, but not a significant change; two points = moderate, i.e., significant, but not a dramatic change; three points $=$ severe, i.e., very marked or prominent, a dramatic change). The maximum score on the severity subscale was thus 12 symptoms x 3 maximum points per symptom $=36$ points. Note that the mean score of patients on the delusions subscale was $0.31(S D=0.14)$, the mean score on the hallucinations subscale was 0.19 $(S D=0.08)$, the mean score on the agitation subscale was $0.50(S D=0.21)$, the mean score on the depression subscale was $0.45(S D=0.18)$, the mean score on the anxiety subscale was $0.20(S D=0.09)$, the mean score on the euphoria subscale was $0.00(S D=0.00)$, the mean score on the apathy subscale was 0.65 $(S D=0.29)$, the mean score on the disinhibition subscale was $0.14(S D=0.06)$, the mean score on the irritability subscale was $0.51(S D=0.21)$, the mean score on the aberrant motor activity subscale was $0.25(S D=0.11)$, the mean score on the night-time behavior disturbance subscale was $0.08(S D=0.04)$, and the mean score on the appetite disorders subscale was $0.04(S D=0.01)$.

Caregivers were also invited to rate the distress they experience due to each symptom on a five-points scale $($ zero $=$ not distressing at all; one $=$ minimal, i.e., slightly distressing, not a problem to cope with; two $=$ mild, i.e., not very distressing, generally easy to cope with; three = moderate, i.e., fairly distressing, not always easy to cope with; four $=$ severe, i.e., very distressing, difficult to cope with; five = very severe, i.e., extremely distressing, unable to cope with). The 
maximum score on the severity subscale was thus 12 symptoms $\times 5$ maximum points per symptom $=60$ points.

\section{Statistical analysis}

To tests our hypotheses, we first compared the number of patients who had changes in neuropsychiatric symptoms during the confinement (i.e., those whose caregivers reported that they had noticed behavioral or emotional changes, either new symptoms or worsening of pre-existing symptoms, during, compared with before, the confinement) versus those who did not. For patients who had changes in neuropsychiatric symptoms during the confinement, we computed Spearman's rank correlations between scores on the severity/distress subscales of the NPIQ and the duration of confinement, i.e., number of days from the beginning of confinement to the date of the interview. Finally, we compared performance scores on the Mini-Mental State Examination between patients in the two groups from their last Memory Center visit. We used Welch- $t$ test due to the unequal effect size. We provided effect size $[d=0.2$ can be considered a small effect size, $d=0.5$ represents a medium effect size and $d=0.8$ refers to a large effect size [28]]. The effect size for Chi square tests was calculated for non-parametric tests following recommendations by Rosenthal and DiMatteo [29], and Ellis [30]. For all tests, level of significance was set as $p \leq 0.05$.

\section{RESULTS}

Among the 38 AD patients, only 10 presented neuropsychiatric changes during the confinement $\left[\chi^{2}(1, N=38)=8.54, p=0.004\right.$, Cohen's $\left.d=1.08\right]$. The mean score on the severity subscale of the NPIQ for these 10 patients was $5.20(S D=4.92)$. The mean score on the caregiver distress subscale for these 10 patients was $5.11(S D=4.99)$. The mean duration of confinement of the $38 \mathrm{AD}$ patients was 27.37 days $(S D=14.48)$. No significant differences were observed between the duration of confinement for the $28 \mathrm{AD}$ patients without neuropsychiatric changes $(M=26.21, S D=15.69)$ and the $10 \mathrm{AD}$ patients with neuropsychiatric changes $(M=30.60, S D=10.40)$, $t(36)=0.82, p=0.42$, Cohen's $d=0.30$. Critically, and as illustrated in Fig. 1, significant positive correlations were observed between the duration of confinement for these $10 \mathrm{AD}$ patients with neuropsychiatric changes and 1) their scores on the severity

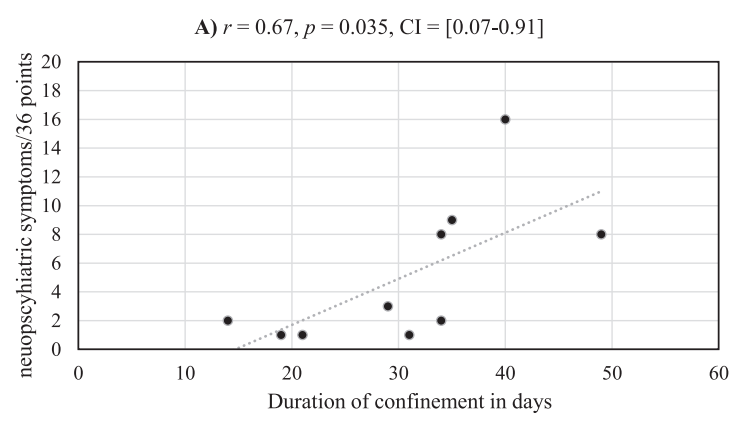

B) $r=0.66, p=0.039, \mathrm{CI}=[0.05-0.91]$

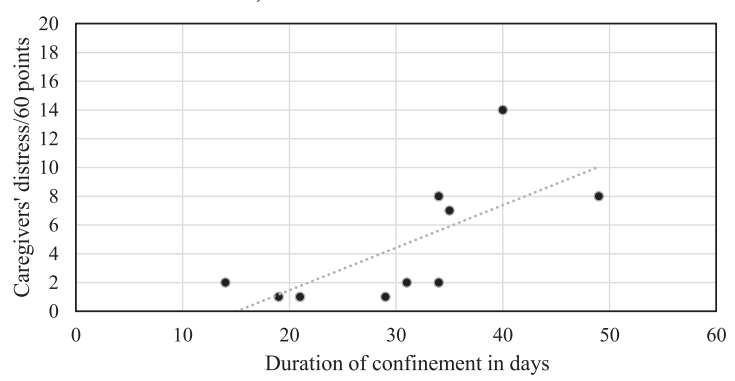

Fig. 1. Medium strength correlations are observed between the duration of confinement and severity of neuropsychiatric symptoms (A) and their care givers' distress (B) for the 10 AD patients who experienced neuropsychiatric changes during the confinement.

subscale of the NPIQ and 2) scores on the caregiver distress subscale of the NPIQ.

Finally, we found that patients who demonstrated neuropsychiatric changes during the confinement had had lower general cognitive functioning at the time of their last Memory Clinic visit,as reflected on MMSE scores, than those who did not demonstrate such changes $(M=15.20, S D=5.14)$ in $(M=20.39$, $S D=6.04), t(18.56)=5.84, p=0.017$.

\section{DISCUSSION}

This is the first study on the effects of confinement during the COVID-19 crisis on neuropsychiatric symptoms in patients with AD. Among our cohort of 38 patients with AD, only 10 had any neuropsychiatric changes during the confinement. For these 10 patients, the duration of confinement significantly correlated with both the severity of their neuropsychiatric symptoms, as well as with the distress experienced by their caregivers. Also, these 10 patients had demonstrated lower cognitive function, in the 2 to 4 months prior to confinement, compared with patients who did not demonstrate any neuropsychiatric changes. 
As demonstrated by our study, confinement resulted in neuropsychiatric changes in a minority of patients with $\mathrm{AD}$. These results are encouraging, as one may have expected that home confinement would have impacted the emotional and behavioral functioning in the majority of patients with AD. Interestingly, no significant differences were over served regarding the duration of confinement between the 10 patients who demonstrated neuropsychiatric changes and that of the 29 patients who did not demonstrate such changes. Thus, the differences between these two populations appear to be intrinsic and to not have been influenced by the duration of confinement. However, the emergence of neuropsychiatric changes in a subset of patients with $\mathrm{AD}$ can be attributed to their degree of cognitive impairment. The 10 patients who demonstrated neuropsychiatric changes had already had lower cognitive functioning compared with patients who did not demonstrate neuropsychiatric changes. Due to greater cognitive decline, AD patients with low cognitive function may be more prone to experience confusion and disorientation, which may have resulted in a greater incidence of neuropsychiatric symptoms during the confinement. The reduced social contact and stimulation and/or reduced physical activity during the confinement might also have induced changes in neuropsychiatric symptoms in these 10 patients. This hypothesis can be supported by the significant positive correlations between the severity of neuropsychiatric symptoms and the duration of confinement. In other words, the longer the confinement, the more severe the symptoms.

For the 10 patients who demonstrated neuropsychiatric changes, the duration of confinement was not only significantly correlated with symptom severity, but also with their care givers' distress. Generally speaking, family caregivers of patients with $A D$, sometimes called the "invisible second patients"[31], are impacted by the quality of life and neuropsychiatric functioning of the patients. Neuropsychiatric symptoms increase distress in caregiver who typically struggle to manage behavioral consequences of these symptoms $[25,26]$. Although caring can be associated with some positive feelings and outcomes, such as reciprocal bonding and feelings of accomplishments and mastery [32], high psychological distress can be experienced by caregivers who are motivated by a sense of guilt, duty or social and cultural norms [33]. Regardless of their motivation, our findings suggest that support should be offered to caregivers by social service authorities during crises, such as the COVID-19 one, to help them cope with their own social isolation as well as the neuropsychiatric changes observed in the patients for whom they care. This support could include instrumental support (e.g., helping them with their daily living needs, housework, and managing neuropsychiatric changes in spouses), emotional support, informational support, and peer support from other caregivers who experience similar challenges.

By demonstrating that effects of confinement on neuropsychiatric function mainly seen among patients with low cognitive performance, our study contributes to the understanding of the underpinning of neuropsychiatric symptoms in AD. The neurological bases of these symptoms have been extensively studied. For instance, hallucinations in AD have been associated with reduced thickness in the lateral parietal cortex, an area of visuospatial integration, [34], increased occipital periventricular hyperintensities, suggesting disruption of visual network connections, [35], as well as with low regional perfusion of the right and left dorsolateral frontal, left ventral striatal, left anterior cingulate, left pulvinar, and dorsolateral parietal cortex regions [36]. Delusions in $\mathrm{AD}$, especially persecutory ones, have been associated with cell loss in the dorsal raphe nucleus, implicating serotonin neurotransmission [37]. Agitation in $\mathrm{AD}$ has been associated with structural and functional abnormalities of brain regions associated with emotional regulation, such as the frontal, anterior cingulate, and posterior cingulate cortices and amygdala [38]. Apathy in AD has been associated with atrophy, hypometabolism, and hypoperfusion in the posterior cingulate, an area involved in motivation and goal-directed behavior, among other regions [39]. Depression in AD has been associated with more severe neuropathology (e.g., higher levels of tau, amyloid- $\beta$ (A $\beta)$, and vascular disease) [40]. Sleep disturbances in AD have been associated with increased accumulation of $A \beta$ in the brain [41]. Taken together, the literature has mainly investigated the neural underpinning of specific neuropsychiatric symptoms in $\mathrm{AD}$ and far less attention has been paid to possible effects of external and social factors on these symptoms. For instance, a study has demonstrated a relationship between loneliness and hallucinations in $\mathrm{AD}$ [42]. More broadly, by demonstrating the effects of confinement on the neuropsychiatric function in patients with low cognitive performance in $\mathrm{AD}$, our study provides evidence on the effects of social factors on neuropsychiatric function in $\mathrm{AD}$. 
Limitations of our study include the relatively small cohort and the lack of neuropsychiatric assessment before the confinement. While the confinement was unpredictable, assessment of neuropsychiatric symptoms before the COVID-19 crisis would have allowed for a better understanding of neuropsychiatric changes in our participants.

To summarize, by offering the first investigation of neuropsychiatric symptoms during the COVID-19 crisis, our study offers a helpful insight into the effects of home confinement on neuropsychiatric function in AD. Critically, our study demonstrates how the confinement exacerbated neuropsychiatric symptoms in patients with low cognitive function in $\mathrm{AD}$, whereas no such symptoms were induced in patients with more preserved cognition.

\section{ACKNOWLEDGMENTS}

DimitriosKapogiannis was supported by the Intramural Research Program of the National Institute on Aging, National Institutes of Health. The study was supported by the LABEX (excellence laboratory, program investment for the future) DISTALZ (Development of Innovative Strategies for a Transdisciplinary Approach to Alzheimer Disease) and the EU CASCADE Interreg 2 Seas Programme 2014-2020 (co-funded by the European Regional Development Fund).

Authors' disclosures available online (https:// www.j-alz.com/manuscript-disclosures/20-0604r1).

\section{REFERENCES}

[1] McKhann G, Knopman DS, Chertkow H, Hyman BT, Jack CR, Jr., Kawas CH, Klunk WE, Koroshetz WJ, Manly JJ, Mayeux R, Mohs RC, Morris JC, Rossor MN, Scheltens P, Carrillo MC, Thies B, Weintraub S, Phelps CH (2011) The diagnosis of dementia due to Alzheimer's disease: Recommendations from the National Institute on Aging-Alzheimer's Association workgroups on diagnostic guidelines for Alzheimer's disease. Alzheimers Dement 7, 263-269.

[2] El Haj M, Antoine P, Amouyel P, Lambert JC, Pasquier F, Kapogiannis D (2016) Apolipoprotein E (APOE) epsilon4 and episodic memory decline in Alzheimer's disease: A review. Ageing Res Rev 27, 15-22.

[3] Lyketsos CG, Carrillo MC, Ryan JM, Khachaturian AS, Trzepacz P, Amatniek J, Cedarbaum J, Brashear R, Miller DS (2011) Neuropsychiatric symptoms in Alzheimer's disease. Alzheimers Dement 7, 532-539.

[4] Bomilcar I, Morris RG, Brown RG, Mograbi DC (2018) Implicit behavioral change in response to cognitive tasks in Alzheimer disease. Cogn Behav Neurol 31, 2-12.
[5] Mograbi DC, Brown RG, Morris RG (2009) Anosognosia in Alzheimer's disease - The petrified self. Conscious Cogn 18, 989-1003.

[6] Lyketsos CG, Sheppard J-ME, Steinberg M, Tschanz JAT, Norton MC, Steffens DC, Breitner JCS (2001) Neuropsychiatric disturbance in Alzheimer's disease clusters into three groups: The Cache County study. Int J Geriatr Psychiatry 16, 1043-1053.

[7] Tschanz JT, Corcoran CD, Schwartz S, Treiber K, Green RC, Norton MC, Mielke MM, Piercy K, Steinberg M, Rabins PV, Leoutsakos JM, Welsh-Bohmer KA, Breitner JC, Lyketsos CG (2011) Progression of cognitive, functional, and neuropsychiatric symptom domains in a population cohort with Alzheimer dementia: The Cache County Dementia Progression study. Am J Geriatr Psychiatry 19, 532-542.

[8] El Haj M, Badcock JC, Jardri R, Larøi F, Roche J, Sommer IE, Gallouj K (2019) A look into hallucinations: The relationship between visual imagery and hallucinations in Alzheimer's disease. Cogn Neuropsychiatry 24, 275-283.

[9] El Haj M, Larøi F (2020) Confabulations on time: Relationship between confabulations and timing deviations in Alzheimer's disease. Arch ClinNeuropsychol 35, 377-384.

[10] Zhao Q-F, Tan L, Wang H-F, Jiang T, Tan M-S, Tan L, Xu W, Li J-Q, Wang J, Lai T-J, Yu J-T (2016) The prevalence of neuropsychiatric symptoms in Alzheimer's disease: Systematic review and meta-analysis. J Affect Disord 190, 264-271.

[11] Lanctôt KL, Amatniek J, Ancoli-Israel S, Arnold SE, Ballard C, Cohen-Mansfield J, Ismail Z, Lyketsos C, Miller DS, Musiek E, Osorio RS, Rosenberg PB, Satlin A, Steffens D, Tariot P, Bain LJ, Carrillo MC, Hendrix JA, Jurgens H, Boot B (2017) Neuropsychiatric signs and symptoms of Alzheimer's disease: New treatment paradigms. Alzheimers Dement (N Y) 3, 440-449.

[12] Rosenberg PB, Mielke MM, Appleby BS, Oh ES, Geda YE, Lyketsos CG (2013) The association of neuropsychiatric symptoms in MCI with incident dementia and Alzheimer disease. Am J Geriatr Psychiatry 21, 685-695.

[13] Peters ME, Schwartz S, Han D, Rabins PV, Steinberg M, Tschanz JT, Lyketsos CG(2015) Neuropsychiatric symptoms as predictors of progression to severe Alzheimer's dementia and death: The Cache County Dementia Progression Study. Am J Psychiatry 172, 460-465.

[14] Dietlin S, Soto M, Kiyasova V, Pueyo M, de Mauleon A, Delrieu J, Ousset PJ, Vellas B (2019) Neuropsychiatric symptoms and risk of progression to Alzheimer's disease among mild cognitive impairment subjects. J Alzheimers Dis 70, 25-34.

[15] Donovan NJ, Amariglio RE, Zoller AS, Rudel RK, GomezIsla T, Blacker D, Hyman BT, Locascio JJ, Johnson KA, Sperling RA, Marshall GA, Rentz DM (2014) Subjective cognitive concerns and neuropsychiatric predictors of progression to the early clinical stages of Alzheimer disease. Am J Geriatr Psychiatry 22, 1642-1651.

[16] Duara R, Loewenstein DA, Greig MT, Potter E, Barker W, Raj A, Schinka J, Borenstein A, Schoenberg M, Wu Y, Banko J, Potter H (2011) Pre-MCI and MCI: Neuropsychological, clinical, and imaging features and progression rates. Am J Geriatr Psychiatry 19, 951-960.

[17] Banks SJ, Raman R, He F, Salmon DP, Ferris S, Aisen P, Cummings J (2014) The Alzheimer's Disease Cooperative Study Prevention Instrument Project: Longitudinal outcome of behavioral measures as predictors of cognitive decline. Dement Geriatr Cogn Dis Extra 4, 509-516. 
[18] Liew TM (2020) Neuropsychiatric symptoms in cognitively normal older persons, and the association with Alzheimer's and non-Alzheimer's dementia. Alzheimers Res Ther 12, 35.

[19] Geda YE, Roberts RO, Mielke MM, Knopman DS, Christianson TJ, Pankratz VS, Boeve BF, Sochor O, Tangalos EG, Petersen RC, Rocca WA (2014) Baseline neuropsychiatric symptoms and the risk of incident mild cognitive impairment: A population-based study. Am J Psychiatry 171, 572-581.

[20] Kales HC, Gitlin LN, Lyketsos CG (2015) Assessment and management of behavioral and psychological symptoms of dementia. BMJ 350, h369.

[21] Masters MC, Morris JC, Roe CM (2015) "Noncognitive" symptoms of early Alzheimer disease: A longitudinal analysis. Neurology 84, 617-622.

[22] Ismail Z, Smith EE, Geda Y, Sultzer D, Brodaty H, Smith G, Agüera-Ortiz L, Sweet R, Miller D, Lyketsos CG (2016) Neuropsychiatric symptoms as early manifestations of emergent dementia: Provisional diagnostic criteria for mild behavioral impairment. Alzheimers Dement 12, 195202.

[23] Fischer CE, Ismail Z, Youakim JM, Creese B, Kumar S, Nunez N, Ryan Darby R, Di Vita A, D’Antonio F, de Lena C, McGeown WJ, Ramit R, Rasmussen J, Bell J, Wang H, Bruneau MA, Panegyres PK, Lanctot KL, Aguera-Ortiz L, Lyketsos C, Cummings J, Jeste DV, Sano M, Devanand DP, Sweet RA, Ballard C (2020) Revisiting criteria for psychosis in Alzheimer's disease and related dementias: Toward better phenotypic classification and biomarker research. $J$ Alzheimers Dis 73, 1143-1156.

[24] Cummings JL, Mega M, Gray K, Rosenberg-Thompson S, Carusi DA, Gornbein J (1994) The Neuropsychiatric Inventory: Comprehensive assessment of psychopathology in dementia. Neurology 44, 2308-2314.

[25] Gonzalez-Salvador MT, Arango C, Lyketsos CG, Barba AC (1999) The stress and psychological morbidity of the Alzheimer patient caregiver. Int J Geriatr Psychiatry 14, 701-710.

[26] Boutoleau-Bretonniere C, Vercelletto M (2009) [Caregiver burden in dementia: Relationships with the activities of daily living, behavioral, and psychological symptoms]. Psychol Neuropsychiatr Vieil 7 Spec No 1, 15-20.

[27] Folstein MF, Folstein SE, McHugh PR (1975) "Mini-mental state". A practical method for grading the cognitive state of patients for the clinician. J Psychiatr Res 12, 189-198.

[28] Cohen J (1988) Statistical power analysis for the behavioral sciences. Erlbaum Associates, Hillsdale, NJ.

[29] Rosenthal R, DiMatteo MR (2001) Meta-analysis: Recent developments in quantitative methods for literature reviews. Annu Rev Psychol 52, 59-82.
[30] Ellis PD (2010) The Essential Guide to Effect Sizes: Statistical Power, Meta-Analysis, and the Interpretation of Research Results. Cambridge University Press, New York, NY.

[31] Brodaty H, Donkin M (2009) Family caregivers of people with dementia. Dialogues Clin Neurosci 11, 217-228.

[32] Cohen CA, Colantonio A, Vernich L (2002) Positive aspects of care giving: Rounding out the caregiver experience. Int $J$ Geriatr Psychiatry 17, 184-188.

[33] Pyke KD, Bengtson VL (1996) Caring more or less: Individualistic and collectivist systems of family eldercare. $J$ Marriage Fam 58, 379-392.

[34] Donovan NJ, Wadsworth LP, Lorius N, Locascio JJ, Rentz DM, Johnson KA, Sperling RA, Marshall GA, Alzheimer Disease Neuroimaging Initiative (2014) Regional cortical thinning predicts worsening apathy and hallucinations across the Alzheimer disease spectrum. Am J Geriatr Psychiatry 22, 1168-1179.

[35] Lin SH, Yu CY, Pai MC (2006) The occipital white matter lesions in Alzheimer's disease patients with visual hallucinations. Clin Imaging 30, 388-393.

[36] Mega MS, Lee L, Dinov ID, Mishkin F, Toga AW, Cummings JL (2000) Cerebral correlates of psychotic symptoms in Alzheimer's disease. J NeurolNeurosurg Psychiatry 69, 167-171.

[37] Forstl H, Burns A, Levy R, Cairns N (1994) Neuropathological correlates of psychotic phenomena in confirmed Alzheimer's disease. Br J Psychiatry 165, 53-59.

[38] Rosenberg PB, Nowrangi MA, Lyketsos CG (2015) Neuropsychiatric symptoms in Alzheimer's disease: What might be associated brain circuits? Mol Aspects Med 43-44, 25-37.

[39] Lanctot KL, Aguera-Ortiz L, Brodaty H, Francis PT, Geda YE, Ismail Z, Marshall GA, Mortby ME, Onyike CU, Padala PR, Politis AM, Rosenberg PB, Siegel E, Sultzer DL, Abraham EH (2017) Apathy associated with neurocognitive disorders: Recent progress and future directions. Alzheimers Dement 13, 84-100.

[40] Holmes C, Arranz M, Collier D, Powell J, Lovestone S (2003) Depression in Alzheimer's disease: The effect of serotonin receptor gene variation. Am J Med Genet B Neuropsychiatr Genet 119B, 40-43.

[41] Kang JE, Lim MM, Bateman RJ, Lee JJ, Smyth LP, Cirrito JR, Fujiki N, Nishino S, Holtzman DM (2009) Amyloidbeta dynamics are regulated by orexin and the sleep-wake cycle. Science 326, 1005-1007.

[42] El Haj M, Jardri R, Laroi F, Antoine P (2016) Hallucinations, loneliness, and social isolation in Alzheimer's disease. Cogn Neuropsychiatry 21, 1-13. 